\begin{tabular}{|c|c|c|c|}
\hline$\bigcup_{\substack{\text { INESEG } \\
\text { INTERNATIONAL }}}$ & $\begin{array}{r}\text { International . } \\
\text { Res } \\
w w y\end{array}$ & $\begin{array}{l}\text { urnal of Health Services, } \\
\text { ch and Policy } \\
\text { rgipark.org.tr/ijhsrp }\end{array}$ & \\
\hline $\begin{array}{l}\text { ENGINEERING, } \\
\text { SCIENCE AND } \\
\text { EDUCATION GROUP }\end{array}$ & e-ISSN: 2602-3482 & DOI:10.23884/ijhsrp.2019.4.2.06 & IJHSRP \\
\hline
\end{tabular}

Research Article

\title{
HISTOLOGICAL OF CHANGES IN THE AMNIOTIC MEMBRANE AND PLACENTAL VILLOUS BASAL LAMINA IN COMPLICATED PREGNANCIES
}

\author{
${ }^{*}$ Yusuf NERGIZ ${ }^{1}{ }^{\text {iD }}$, Engin DEVECI ${ }^{1}{ }^{\text {iD }}$, Erdal SAK $^{2}{ }^{\text {iD }}$, Siddik EVSEN $^{2}{ }^{\text {iD }}$, Cenap EKINCI $^{1}$ \\ , Şebnem NERGIZ ${ }^{3}$ iD , Beyza KARADEDE ${ }^{4}$ (D) Ŭgur ŞEKER ${ }^{1}$ iD \\ ${ }^{1}$ University of Dicle, Faculty of Medicine, Department of Histology and Embryology, Diyarbakır, Turkey. \\ ${ }^{2}$ University of Dicle, Faculty of Medicine, Department of Obstetrics and Gynecology, Diyarbakır, Turkey. \\ ${ }^{3}$ University of Dicle, Atatürk Health School, Diyarbakır, Turkey. \\ ${ }^{4}$ University of Katip Çelebi, Faculty of Dentistry, Department of Orthodontic, İzmir, Turkey. \\ *Corresponding author; yusufnergiz21@gmail.com
}

\begin{abstract}
This study aimed to histological changes compare placental villous basal lamina and amniotic membrane changes in complicated pregnancy. Studies were performed on the human placentas, delivered between 24-39 weeks of gestation. Patients were separated equally into 4 groups (Control, preeclampsia (PE), gestastional diabetes (GD), and HELLP syndrome groups). Placental tissue samples were dissected and fixed in 10\% neutral formalin buffer. Routine paraffin tissue protocol was followed. Some of the sections were stained with Periodic Acid Schiff. Remaining sections were stained with integrin alpha-6 antibody. To define expression percentage, mean of the staining area/total staining area ratio were calculated. The statistical significance of the expression percentages was compared by One Way ANOVA and Tukey tests with SPSS Statistics V24 software. In PAS-stained preeclamptic, HELLP and gestational diabetes groups placental villous basal lamina and vasculosyncytial membranes were thicker than the control group. A significant difference was observed in all 3 groups compared to the control group the placental villous basal lamina thickness of the HELLP group was found to be significantly different from all three groups. In chorionic villi of HELLP group, dense integrin expression was found in placental villous basal lamina similar to that in GD and preeclampsia groups. The HELLP group was significantly different from all groups. In preeclampsia, gestational diabetes and HELLP placentas, the placental viiöz basal lamina and amniotic membrane significantly thickened and structural changes were observed.
\end{abstract}

Key Words: Placenta, Basal lamina, Preeclampsia, Gestational diabetes, HELLP syndrome

Received: July 3, $2019 \quad$ Accepted: July 22, 2019

\section{Introduction}

The placenta develops where complex and coordinated interactions undergo between fetal embryonic tissue and pregnant endometrial tissues. Throughout the pregnancy, even on the 10th day of pregnancy, a large number of placental macrophages were detected in the placenta [1]. Placental macrophages consist of Hofbauer cells in the fetal chorionic villi and decidual macrophages in the maternal decidua basalis [2]. Among the functions, placental macrophages produce factors that regulate local immune reactions (factors that regulate maternal immunological tolerance and protect the fetus) and stimulate placental angiogenesis during placental development [3]. The integrin alpha 6/beta 4 complex is a member of the integrin family of adhesion receptors. It is found in a variety of epithelial cell types but is most strongly expressed in stratified squamous epithelia. It is a receptor for laminin in epithelial cells and it plays a critical structural role in the hemidesmosome. The alpha 6 subunit is also strongly localized to the basal region but also, is present over the entire surfaces of basal cells and 
some cells in the immediate suprabasal region. Localization is generally cell surface and hemidesmosome [4].

This study aimed to histological changes compares placental villous basal lamina and amniotic membrane changes in complicated pregnancy.

\section{Materials and methods}

The Ethics Committee of Research Center of Dicle University Hospital approved the protocol. Studies were performed on the human placentas, delivered between 24-39 weeks of gestation. Patients were divided equally into 4 groups (Control, preeclampsia, gestastional diabetes, and HELLP syndrome groups) with a total of 40 full-term human placentas. All women recruit were singleton pregnancy, between 24- 39 weeks of gestastional age. These patients were aged between 20 and 37 years (mean \pm SD: $27.2 \pm 3.1$ years). The birth weight of the 40 live births was $1850-3300$ grams. The average placenta weight of control group was $672 \mathrm{~g}$, PE group $387 \mathrm{~g}$, HELLP group $340 \mathrm{~g}$, and in GD group $491 \mathrm{~g}$ respectively. For grouping, the World Health Organization (WHO) criteria were considered:

Diagnostic criteria for preeclampsia are systolic blood pressure $\geq 140 \mathrm{mmHg}$ or diastolic blood pressure $\geq 90 \mathrm{mmHg}$ after 20 weeks of gestation. Proteinuria $\geq 0.3 \mathrm{~g}$ in a 24-hour urine specimen.

In the Tennessee Classification System diagnostic criteria for HELLP (hemolysis, elevated liver enzymes, and low platelet count) are hemolysis with increased LDH ( $>600 \mathrm{U} / \mathrm{L})$, AST ( $\geq 70 \mathrm{U} / \mathrm{L})$, and platelets $<100,000$ cells $/ \mathrm{mm}$.

Criteria for diabetes mellitus in pregnancy should be diagnosed with fasting plasma glucose $7.0 \mathrm{mmol} / \mathrm{L}$ $(126 \mathrm{mg} / \mathrm{dL})$.

Placental tissue samples were dissected and fixed in $10 \%$ neutral formalin buffer. Routine paraffin tissue protocol was followed. $5 \mu \mathrm{m}$ paraffin sections were stained with Periodic Acid Schiff (PAS). In these sections, the thickness of the plasental villous basal lamina and the amniotic membrane was measured by ocular micrometer and the differences between the groups were evaluated. Remaining sections were stained for immunohistochemistry. To evaluate the integrin expression level of basal membrane, sections were incubated with anti-integrin alpha-6 antibody (cat no: ab133386, dilution rate: 1/750, abcam). 5 areas were randomly chosen where the terminal villi were densely stained. To define expression percentage, mean of the staining area/total staining area ratio were calculated. The statistical significance of the expression percentages was compared by One Way ANOVA and Tukey tests with SPSS Statistics V24 software (IBM, USA) and $\mathrm{p}<0.05$ was considered statistically significant. Samples were evaluated by Zeiss Imager A2 light microscope and micrographed.

\section{Results}

3.1. Histochemical Findings: In PAS-stained preeclamptic, help and gestational diabetes group placentas Placental villous basal lamina and vasculo-syncytial membranes were thicker than the control group (Figure-1). In some sections, there were amniotic membrane abnormalities observed in preeclampsia and HELLP group samples (Figure-2).

Placental villous basal lamina thickness measured by 400x magnification in the microscope is shown in Table-1. According to the One-Way Anova, Post-hoc Tukey tests, Placental villous basal lamina thickness of the control group was found to be $1.35 \pm 0.24 \mu \mathrm{m}$. A significant difference was observed in GD and PE groups ( $\mathrm{p}<0.05$ ) compared to the control group. There was no significant difference in Placental villous basal lamina thickness of GD group $(2.02 \pm 0.13 \mu \mathrm{m})$ and preeclampsia group $(1.99 \pm 0.14 \mu \mathrm{m})(\mathrm{p}>0.05)$. The placental villous basal lamina thickness of the HELLP group was 
found to be $2.70 \pm 0.44 \mu \mathrm{m}$ and found to be significantly different from GD and preeclampsia groups ( $\mathrm{p}$ $<0.05)$ and control group $(\mathrm{p}=0.00)$.

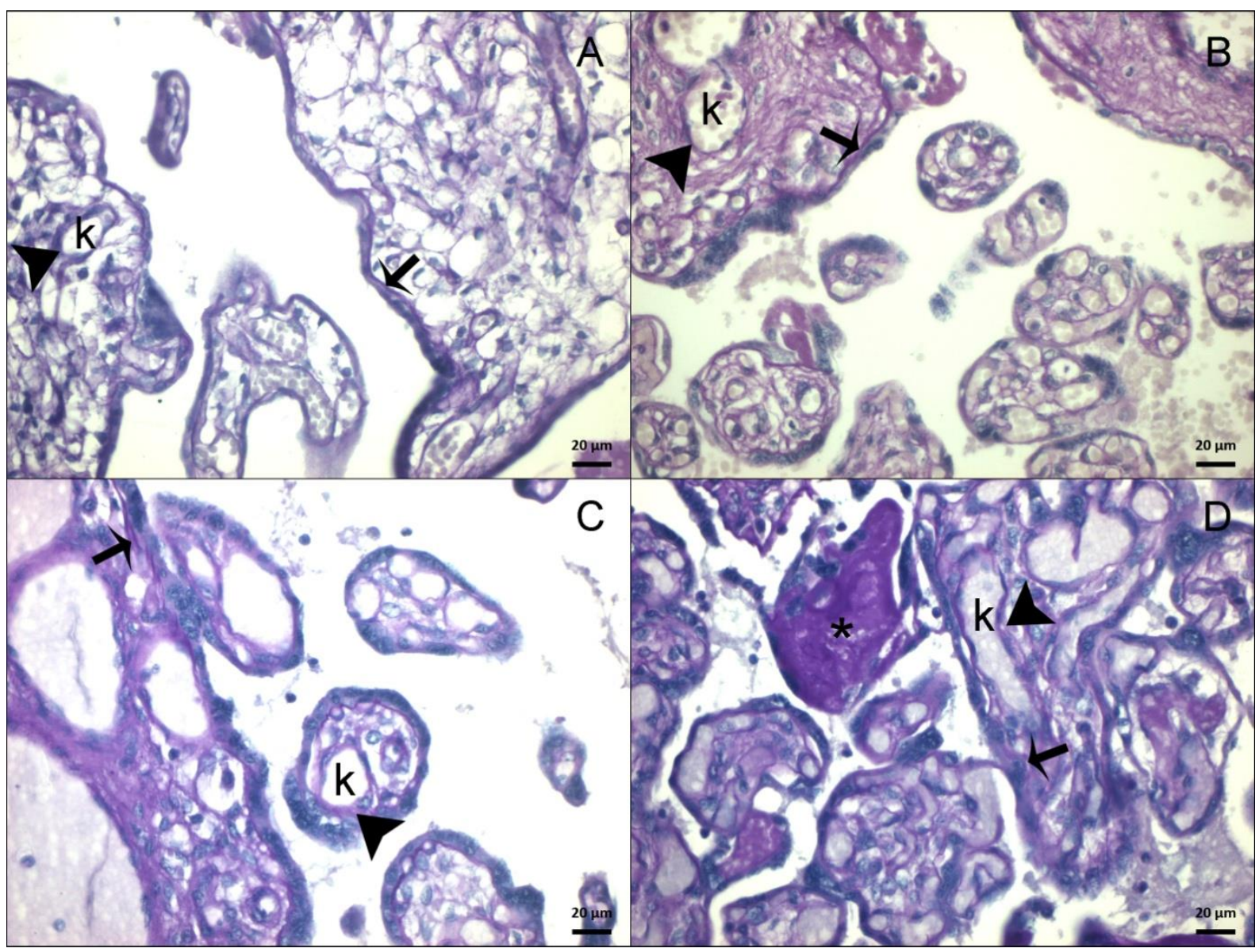

Figure-1: Placental sections stained with PAS. Note thickening of the placental villous basal lamina. A) Control group, B) Preeclampsia group, C) Gestational diabetes, D) HELLP group. Arrow: placental villous basal lamina. Arrowhead: Capillary endothelial basal lamina, asteriks: Fibrin, k: Capillary, (PAS, Bar: $20 \mu \mathrm{m}$ ).

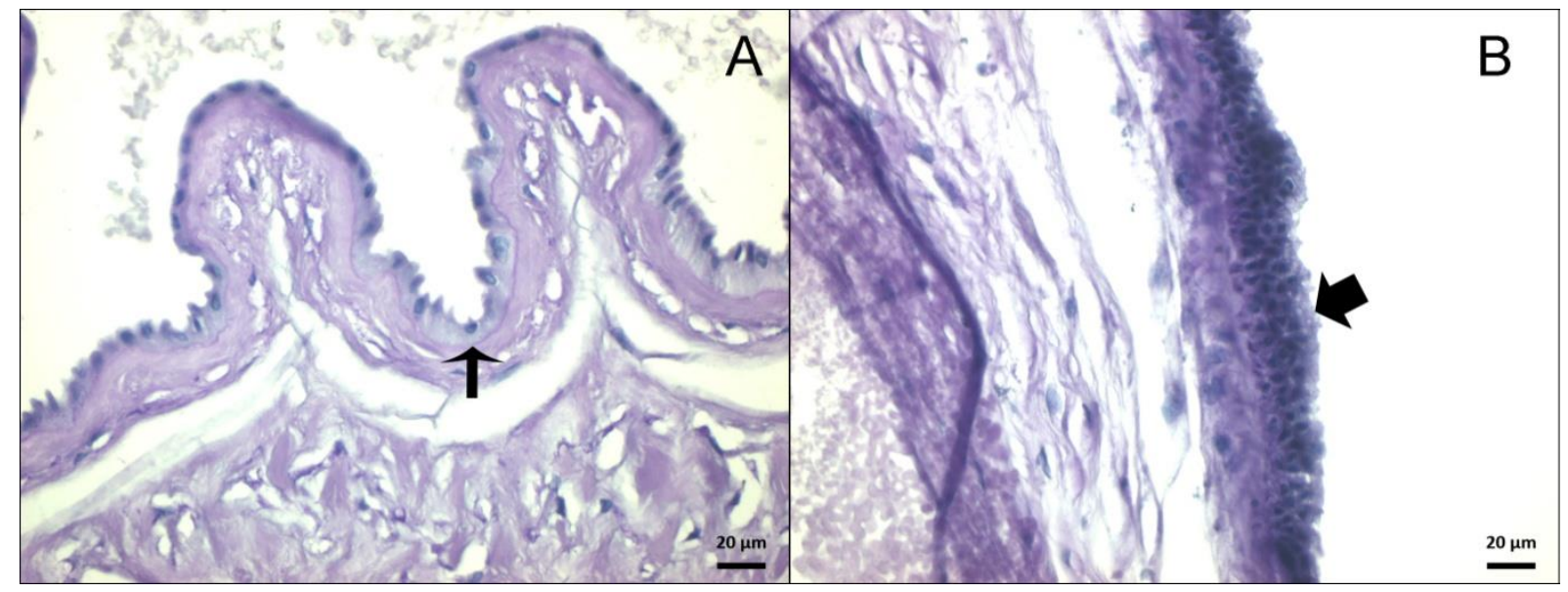

Figure-2: Thickening of the amniotic membrane and squamous metaplasia in the epithelium. A) Preeclampsia group, B) HELLP group, thin arrow: Amniotic membrane, thick arrow: Squamous metaplasia (PAS, Bar $20 \mu \mathrm{m}$ ). 
Table 1: Placental villous basal lamina thickness and Standard Deviation. Values with different superscripts in the same column are significantly different (a-b: $p<0.05$, b-c: $p<0.05$, a-c: $p=0.00$ ).

\begin{tabular}{lccc}
\hline Groups & Mean $(\boldsymbol{\mu m}) \pm$ S.D. & Maximum $(\boldsymbol{\mu m})$ & Minimum $(\boldsymbol{\mu m})$ \\
\hline Control & $1,35 \pm 0,24^{\mathrm{a}}$ & 1,92 & 1,06 \\
\hline GD & $2,02 \pm 0,13^{\mathrm{b}}$ & 2,20 & 1,79 \\
\hline PE & $1,99 \pm 0,14^{\mathrm{b}}$ & 2,17 & 1,73 \\
\hline HELLP & $2,70 \pm 0,44^{\mathrm{c}}$ & 3,54 & 2,14 \\
\hline
\end{tabular}

3.2. Immunohistochemical findings: We observed that integrin is expressed in various areas in control, PE, (GD) and HELLP groups. In the control group, while expression level was normal in the placental villous basal lamina, we observed increased expression in the placental villous basal lamina of GD and PE groups. In chorionic villus of HELLP group, dense integrin expression was found in placental villous basal lamina similar to that in GD and PE groups. Additionally, integrin expression was detected in the basal membrane of capillaries of all groups. Microscopic evaluation revealed that GD, PE, and HELLP groups had more intense expression levels in vasculo-syncytial membranes (Figure-3).

According to the results of the One Way ANOVA, Post-hoc Tukey tests of the expression analysis performed with the Image $\mathrm{J}$ software: The control group basal membrane integrin expression was 16.20 $\pm 2.10 \%$ in the randomly selected areas and the expression area in the GD and PE groups $(\mathrm{p}<0.05)$ and HEELP group $(p=0,00)$ observed significantly different. \%29,60 1,71 GD group and $\% 27,30 \pm 2,21$ PE group were similar expression area $(\mathrm{p}>0.05)$. It was determined that the $\% 35,30 \pm 2,54$ expression area of the HELLP group was significantly different from GD and PE groups $(\mathrm{p}<0.05)$ and control group $(\mathrm{p}=0,00)($ Table-2). 


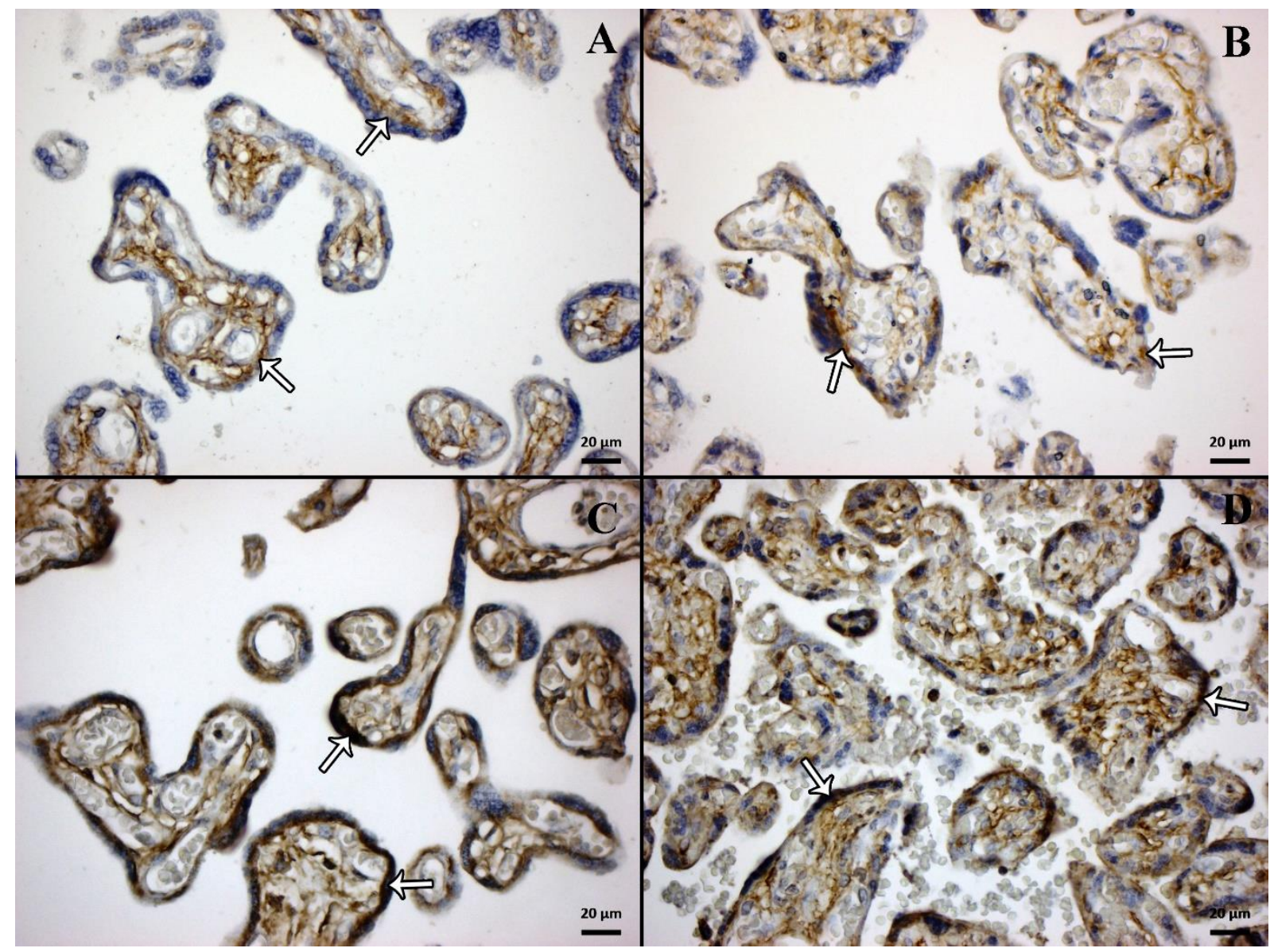

Figure-3: Placental sections stained with integrin alpha-6 antibody. While normal levels of integrin expression (arrow) were observed in the control group, it was increased in expression areas (arrow) of GD, preeclampsia and HELLP groups. A: Control group, B: Preeclampsia group, C: Gestasyonal diabetes group, D: HELLP group (Bar: $20 \mu \mathrm{m}$, Counterstain: Mayer Hematoxylin, integrin alpha-6).

Table 2: Covered area of integrin expression. Values with different superscripts in the same column are significantly different (a-b: $\mathrm{p}<0.05$, b-c: $\mathrm{p}<0.05$, a-c: $\mathrm{p}=0.00)$.

\begin{tabular}{lccc}
\hline Groups & Mean (\%) \pm S.D & Maximum (\%) & Minimum (\%) \\
\hline Control & $19,20 \pm 2,10^{\mathrm{a}}$ & 22,00 & 16,00 \\
\hline GD & $27,60 \pm 1,71^{\mathrm{b}}$ & 30,00 & 24,00 \\
\hline PE & $27,30 \pm 2,21^{\mathrm{b}}$ & 31,00 & 23,00 \\
\hline HELLP & $35,30 \pm 2,54^{\mathrm{c}}$ & 41,00 & 32,00 \\
\hline
\end{tabular}

\section{Discussion}

The placenta is an essential organ for the exchange of nutrients and metabolites between the mother and the fetus. The thickness of the placenta depends on the length of the stem villi [5] Placenta develops in two phases: hyperplasia and subsequent hypertrophy [6-8]. Fetal hyperglycemia may disrupt the osmotic environment leading to cell damage and cell death [9]. This process also involves capillary endothelium. Because the damaged capillary endothelium is present in the former basal laminae, the endothelium regenerates itself [10]. As the newly formed endothelial cells synthesize their basal 
laminae, the endothelial basal laminae of the fetal capillaries in the chorionic villi become extremely thick. Since chorionic capillary basal lamina is a part of the Placental villous basal lamina, it makes placental basal membrane thickened. This thickening means the reduction of oxygen and nutrient transport to the fetus. The organism responds with an increase in placental weight with hyperplasia and basal lamina thickening in terminal villi. In a study by Salvatore et al. [11], Placental villous basal lamina of normotensive placentas was considerably thicker compared to HELLP and diabetic group placentas. Our study supports this evidence. Normal fetal development and growth depend on the function of the placenta. Some forms of intrauterine growth retardation result from placental blood flow failure and insufficient transport of nutrients from mother to fetus [12]. In this study, significantly increased cell hyperplasia and placental villous basal lamina were observed in preeclamptic, HELLP and gestational diabetic placentas (Figure-1) but not observed in placentas of the control group. The Placental villous basal lamina thickness may be the result of the accumulation of mucopolysaccharides. This condition occurs as a result of intrauterine growth retardation and uteroplacental circulation insufficiency [13]. Also, there was a significant thickening of the vasculo-syncytial membranes of the gestational diabetic group and this may be due to the increase in the diffusion distance between mother and fetus blood [12].

In our study, we observed Placental villous basal lamina and vasculo-syncytial basal membrane thickness of placentas in all group except for the control group, congestion in intervillous space, villous edema and thinning in a decidual basal membrane. These findings are similar to previous studies [15]. Placental villous basal lamina and amniotic membrane have significantly thickened and underwent structural changes in groups of preeclampsia, GD and HELLP groups.

In conclusion, in preeclampsia, gestational diabetes, and HELLP syndrome placentas, Placental villous basal lamina and amniotic membrane significantly thickened and structural changes were observed.

\section{Acknowledgment}

This study was supported by the Research Fund of Dicle University, Project No: 13-TF-158.

\section{References}

[1] Chang MDY, Pollard JW, et al. "Mouse placental macrophages have a decreased ability to present antigen", Proceedings of the National Academy of Sciences, 90, 462-466, 1993.

[2] Bulmer JN, Johnson PM. "Macrophage populations in the human placenta and amniochorion", Clinical Experimental Immunology, 57, 393-403, 1984.

[3] Mues B, Langer D, et al. "Phenotypic Characterization of Macrophages in Human Term Placenta", Immunology, 67, 303-307, 1989.

[4] Sonnenberg A, Linders CJ, et al. "The alpha 6 beta 1 (VLA-6) and alpha 6 beta 4 protein complexes: tissue distribution and biochemical properties", J. Cell Sci, 96, 207-217, 1990.

[5] Moore KL., The developing human. 3rd ed., WB Saunders, Philadelphia, 1983.

[6] Laga EM, Dirscoll SG,et al, "Quantitative studies of human placenta", Biol Neonate, 23, 260-283, 1993.

[7] Teasudale F., "Gestational changes in the function structure of human placenta in relation to fetal growth", Am J Obstet Gynec, 137, 560-568, 1980.

[8] Winick M, Coscia A, et al., "Cellular growth in normal placenta”, Pediatr, 39, 248-51, 1987.

[9] Kumar V, Cotran S, et al., Basic pathology, 6th ed., WB Saunders, Pennsylvania, 2000. 
[10] Feczko JD, Kluber KM., "Cytoarchitecture of muscle in genetic model of murine diabetes", Am J Anat, 182, 224-240, 1988.

[11] Salvatore AC., "The placenta in acute toxemia", Am J Obstet. Gynecol, 102, 347-353, 1968.

[12] Honda M, Toyoda C, et al., "Quantitative investigations of placental terminal villi in maternal diabetes mellitus by scanning and transmission electron microscopy”, Tohoku J Exp Med, 167, 247257, 1992.

[13] Benirschke K, Kaufmann P, Baergen RN., Pathology of the human placenta. 6th ed., Springer, Berlin, 2006.

[14] Gewolb I, Merdian W, et al., "Fine structural abnormalities of the placenta in diabetic rats", Diabetes, 35, 254-261, 1986.

[15] Pietryga M, Biczysko W, et al., "Ultrastructural examination of the placenta in pregnancy complicated by diabetes mellitus”, Ginekol Pol, 75,111-118, 2004. 